\title{
Magnetovac cylinder to magnetovac torus
}

\author{
E N Glass \\ Department of Physics, University of Michigan, Ann Arbor, MI 48109, USA
}

Received 10 August 2006

Published 8 November 2006

Online at stacks.iop.org/CQG/23/7455

\begin{abstract}
A method for mapping known cylindrical magnetovac solutions to solutions in torus coordinates is developed. Identification of the cylinder ends changes topology from $R^{1} \times S^{1}$ to $S^{1} \times S^{1}$. An analytic Einstein-Maxwell solution for a toroidal magnetic field in tori is presented. The toroidal interior is matched to an asymptotically flat vacuum exterior, connected by an Israel boundary layer.
\end{abstract}

PACS numbers: 04.20.Jb, 04.40.Dg, 04.40.Nr

\section{Introduction}

Magnetic fields can be found in many astrophysical configurations. Both poloidal and toroidal fields can model the observed dipolar fields in magnetic white dwarfs [1]. The radius of a nonrotating white dwarf can change significantly [2] when a magnetic field is frozen-in. The equilibrium configuration of magnetized stars with both poloidal and toroidal magnetic fields has been studied for some time [3]. It is known that toroidal $\vec{B}$ fields deform neutron stars and it has been shown [4] this deformation acts as a source of gravitational radiation.

Because of the general interest in astrophysical magnetic fields, this work provides a method for mapping known cylindrical magnetovac solutions to magnetovac solutions in nested tori. The cylindrical solution used as an example has an axially symmetric magnetic field which is mapped to tori with a toroidal magnetic field. The cylinder solution is unbounded in distance from the cylinder axis. It is straight forward to map a portion of this solution to a set of tori. If we conclude the map with an unbounded set of tori, nothing further is required. But if the torus solution is matched to an exterior vacuum at a finite boundary, then the metric and extrinsic curvatures, as well as the magnetic field must also satisfy boundary conditions. The magnetic field requires a surface current density, which must reside in an Israel boundary layer [6]. We construct such a layer between the magnetic tori and the exterior vacuum.

The new solution has four distinct regions. The innermost region is a singular loop. The next region extends from the singularity to the vacuum boundary. This second region contains a toroidal magnetic field in nested tori. At the vacuum boundary there is a third region of zero thickness with a delta function current density supported by an Israel layer. The fourth region is an asymptotically flat vacuum. 
The next section discusses the map from cylinders to tori and gives details of the magnetovac cylinder solution. The cylinder solution has a singular $z$-axis. This is mapped to a singular cylinder in torus coordinates. Section 3 gives a new solution for a toroidal magnetic field in torus coordinates. It is shown that the Rainich conditions for an Einstein-Maxwell solution are satisfied. In section 4 the boundary and extrinsic curvatures are presented. Section 5 treats the magnetic field junction conditions, the boundary current, and the stressenergy content of the Israel layer. In section 6 the electromagnetic energy, Komar mass and sectional curvature mass are computed. The sectional curvature mass definition has been extended from spheres to include tori. We show that the magnetic energy subtracts quasilocally from the sectional curvature mass. Section 7 presents the complete metric and its asymptotic and inner regions. The vacuum region is asymptotically flat and can be compactified. We close with a summary. Details are collected in four appendices: appendix A discusses Euclidean torus coordinates. We use coordinates $(r, \alpha, \beta) ; r$ labels successive tori and $(\alpha, \beta)$ are coordinates on a single torus [5]. Appendix B analyses geodesics in the plane surrounding the singular region by examining timelike geodesics paths in an effective potential. Appendix $\mathrm{C}$ covers torus null tetrad quantities. Appendix D lists electromagnetic field equations and the Rainich conditions.

Conventions. Riemann and Ricci sign conventions are $2 A_{\nu ;[\alpha \beta]}=A_{\mu} R_{\nu \alpha \beta}^{\mu}$ and $R_{\alpha \beta}=R^{\nu}{ }_{\alpha \beta \nu}$. The metric signature is $(+,-,-,-)$ and the field equations are $G_{\mu \nu}=-8 \pi T_{\mu \nu}$. We use units such that $G=c=1$.

Magnetic field terminology. In cylindrical or spherical coordinate systems, magnetic field $\vec{B}$ has orthogonal toroidal and poloidal components. A component is toroidal if it is everywhere orthogonal to meridional planes through the cylinder axis or planes through the spherical poles. If $\vec{B}$ has axial symmetry, toroidal field lines are circles about the cylinder axis or spherical circles of longitude. A component lying totally in meridional planes is poloidal, and is orthogonal to the toroidal component.

\section{Cylinder to torus}

A torus is the only compact, oriented, Euler number zero, two-dimensional surface. A cylinder can be made into a torus by joining its ends.

The cylindrical 3-metric is $\mathrm{d} \tilde{r}^{2}+\tilde{r}^{2} \mathrm{~d} \varphi^{2}+\mathrm{d} z^{2}$, and this is mapped to torus coordinates as $\mathrm{d} r^{2}+r^{2} \mathrm{~d} \beta^{2}+\Re^{2} \mathrm{~d} \alpha^{2}$. (Details of torus coordinates are given in appendix A.) Cylindrical $\tilde{r} \rightarrow$ torus $r$, and cylindrical $\varphi \rightarrow$ torus $\beta$. For the cylinder ends, $\mathrm{d} z=\Re \mathrm{d} \alpha$. Since $-\infty<z<\infty$ and $0 \leqslant \alpha \leqslant 2 \pi$, the cylinder is wrapped to a torus. This identification takes $R^{1} \times S^{1}$ to $S^{1} \times S^{1}$. The cylinder $z$-axis corresponds to $\Re=r_{0}+r \cos \beta=0$. Because of torus topology, flat metric $\mathrm{d} t^{2}-\left(\mathrm{d} r^{2}+r^{2} \mathrm{~d} \beta^{2}+\mathfrak{R}^{2} \mathrm{~d} \alpha^{2}\right)$ is not the Minkowksi metric.

Cylinder solution. The cylindrical metric we consider (equation (22.11), $m=2$, in Exact Solutions [7]) is

$g_{\mu \nu}^{\text {cyl }} \mathrm{d} x^{\mu} \mathrm{d} x^{\nu}=\tilde{r}^{4}\left(F / b_{0}\right)^{2}\left(\mathrm{~d} t^{2}-\mathrm{d} \tilde{r}^{2}\right)-\tilde{r}^{-2}\left(F / b_{0}\right)^{2} \mathrm{~d} \varphi^{2}-\tilde{r}^{4}\left(b_{0} / F\right)^{2} \mathrm{~d} z^{2}$,

with $F(\tilde{r})=1+b_{0}^{2} \tilde{r}^{4}$. This solution satisfies the Einstein-Maxwell field equations and has an axially symmetric magnetic field along the $z$-axis. The Kretschmann scalar is

$$
R^{\alpha \beta \mu \nu} R_{\alpha \beta \mu \nu}=64 \frac{b_{0}^{4}\left(3+12 b_{0}^{2} \tilde{r}^{4}+62 b_{0}^{4} \tilde{r}^{8}-108 b_{0}^{6} \tilde{r}^{12}+63 b_{0}^{8} \tilde{r}^{16}\right)}{\tilde{r}^{12} F^{8}} .
$$

Metric $g^{\text {cyl }}$ has a singularity along the $z$-axis, $\tilde{r}=0$, where the Kretschmann scalar becomes infinite. 
The Ricci tensor has components $t, \tilde{r}, \varphi, z$ :

$$
R_{\beta}^{\alpha}=16 \frac{b_{0}^{4}}{\tilde{r}^{2} F^{4}}\left[\begin{array}{cccc}
1 & & & \\
& -1 & & \\
& & 1 & \\
& & & -1
\end{array}\right] \text {. }
$$

The magnetic field is

$$
B_{\mu} \mathrm{d} x^{\mu}=4 \frac{b_{0}^{2}}{\tilde{r}^{2} F} \mathrm{~d} \varphi
$$

with electromagnetic invariants

$$
\frac{1}{2} F_{\mu \nu} F^{\mu \nu}=16 \frac{b_{0}^{4}}{\tilde{r}^{2} F^{4}} \quad \frac{1}{2} F^{\mu v} F_{\mu \nu}^{*}=0 .
$$

The metric and magnetic field can be mapped, locally, to a different metric and magnetic field in a frame of nested tori. After the transform, the resulting metric is $g^{\text {tor }}$ given in equation (4).

\section{Torus solution}

The toroidal magnetic metric is, with a map of cylinder coordinates $\{\tilde{r}, z, \varphi\}$ to torus coordinates $\{r, \alpha, \beta\}$, and with $\Re=r_{0}+r \cos \beta$ and $\digamma=1+b_{0}^{2} \Re^{4}$,

$$
\begin{aligned}
g_{\mu \nu}^{\text {tor }} \mathrm{d} x^{\mu} \mathrm{d} x^{\nu}= & \digamma^{2} \mathfrak{R}^{4} \mathrm{~d} t^{2}-\mathfrak{R}^{4}\left(\digamma^{2} \cos ^{2} \beta+\digamma^{-2} \sin ^{2} \beta\right) \mathrm{d} r^{2}-\digamma^{2} \Re^{-2} \mathrm{~d} \alpha^{2} \\
& +\mathfrak{R}^{4}\left(\digamma^{2}-\digamma^{-2}\right) r \cos \beta \sin \beta 2 \mathrm{~d} r \mathrm{~d} \beta-\mathfrak{R}^{4}\left(\digamma^{2} \sin ^{2} \beta+\digamma^{-2} \cos ^{2} \beta\right) r^{2} \mathrm{~d} \beta^{2}
\end{aligned}
$$

with volume element

$$
\left(r \Re^{5} \digamma^{2}\right) \mathrm{d} t \wedge \mathrm{d} r \wedge \mathrm{d} \alpha \wedge \mathrm{d} \beta .
$$

Note that if parameter $p_{0}$ is introduced by $\digamma \rightarrow \digamma / p_{0}$ in metric $g^{\text {tor }}$, just as parameter $b_{0}$ appears in the cylinder metric, then the Ricci and Riemann tensors are multiplied by $p_{0}^{2}$. The limit $p_{0} \rightarrow 0$ takes $g^{\text {tor }}$ to flatness (but not to the Minkowski metric).

The Kretschmann scalar for $g^{\text {tor }}$ is $R_{\alpha \beta \mu \nu} R^{\alpha \beta \mu \nu} \sim 1 / \Re^{12}$. The singularity of $g^{\text {cyl }}$ is mapped to a singular loop, $\Re=0$, at the centre of the torus solution.

Killing symmetries are static $\partial_{t}$ and axial $\partial_{\alpha}$. Constant time hypersurfaces for static $g^{\text {tor }}$ have unit vector

$$
\hat{t}_{\text {tor }}^{\mu} \partial_{\mu}=\digamma^{-1} \Re^{-2} \partial_{t} .
$$

In torus coordinates, the Ricci tensor has components $(t, r, \alpha, \beta)$

$$
R^{\mu}{ }_{\nu}=16 \frac{b_{0}^{2}}{\mathfrak{R}^{2} \digamma^{4}}\left[\begin{array}{llll}
1 & & & \\
& -1 & & \\
& & 1 & \\
& & & -1
\end{array}\right] .
$$

Static metrics have the timelike Killing vector as an eigenvector of the Ricci tensor. For $g^{\text {tor }}$ we find

$$
R^{\mu}{ }_{\nu} \delta_{(t)}^{\nu}=\left(16 \frac{b_{0}^{2}}{\mathfrak{R}^{2} \digamma^{4}}\right) \delta_{(t)}^{\mu}
$$

with eigenvalue twice the magnetic energy density. 
The metric is expanded in a null basis, $g_{\mu \nu}^{\text {tor }}=l_{\mu} n_{\nu}+n_{\mu} l_{\nu}-m_{\mu} \bar{m}_{\nu}-\bar{m}_{\mu} m_{\nu}$ (see appendix B). The only non-zero Ricci component is

$$
\Phi_{11}=8 b_{0}^{2} \Re^{-2} \digamma^{-4} \text {. }
$$

With Ricci tensor

$$
R_{\mu \nu}^{\mathrm{tor}}=-2 \Phi_{11}\left(l_{\mu} n_{v}+n_{\mu} l_{\nu}+m_{\mu} \bar{m}_{\nu}+\bar{m}_{\mu} m_{\nu}\right)
$$

the Ricci 'square' is

$$
R_{\alpha}^{\mu} R_{v}^{\alpha}=4\left(\Phi_{11}\right)^{2} g_{v}^{\mu}
$$

and with $\Phi_{11}$ in equation (8) this becomes

$$
R^{\mu \nu} R_{\mu \nu}=\left(32 \frac{b_{0}^{2}}{\mathfrak{R}^{2} \digamma^{4}}\right)^{2} .
$$

Equation (10) shows the Ricci 'square' satisfies Rainich condition (D.14b).

The vacuum limit, $b_{0} \rightarrow 0$, is

$$
g_{\mu \nu}^{\text {tor-vac }} \mathrm{d} x^{\mu} \mathrm{d} x^{\nu}=\Re^{4} \mathrm{~d} t^{2}-\Re^{4} \mathrm{~d} r^{2}-\Re^{-2} \mathrm{~d} \alpha^{2}-r^{2} \Re^{4} \mathrm{~d} \beta^{2} .
$$

$g^{\text {tor-vac }}$ has zero Ricci tensor and non-zero Riemann tensor. $\left(R_{\alpha \beta \mu \nu} R^{\alpha \beta \mu \nu}\right)_{\text {tor-vac }}=192 / \Re^{12}$.

Electromagnetic field. The vector potential for the Maxwell field is

$$
A_{\mu}^{\text {tor }} \mathrm{d} x^{\mu}=b_{0} \frac{\Re^{4}}{\digamma}(\sin \beta \mathrm{d} r+r \cos \beta \mathrm{d} \beta),
$$

with

$$
F_{\mu \nu}^{\mathrm{tor}}=4 b_{0}\left(\frac{r \Re^{3}}{\digamma^{2}}\right)\left[\delta_{\mu}^{(r)} \delta_{\nu}^{(\beta)}-\delta_{\mu}^{(\beta)} \delta_{\nu}^{(r)}\right]
$$

There is no electric field since $F_{\mu \nu}^{\text {tor }}$ has no time component and there is no current since $\nabla_{\nu} F_{\text {tor }}^{\mu \nu}=0$. The dual Maxwell field is

$$
F_{\mu \nu}^{*}=2 b_{0}\left[\delta_{\mu}^{(\alpha)} \delta_{\nu}^{(t)}-\delta_{\mu}^{(t)} \delta_{\nu}^{(\alpha)}\right] \text {. }
$$

The local magnetic field $B_{\mu}^{\text {tor }}=F_{\mu \nu}^{*} \hat{t}_{\text {tor }}^{v}$, which satisfies $\nabla_{\mu} B^{\mu}=0$, is

$$
B_{\mu}^{\text {tor }} \mathrm{d} x^{\mu}=4 \frac{b_{0}}{\mathfrak{R}^{2} \digamma} \mathrm{d} \alpha, \quad B_{\mu}^{\text {tor }} B_{\text {tor }}^{\mu}=-16 \frac{b_{0}^{2}}{\mathfrak{R}^{2} \digamma^{4}} .
$$

The magnetic field lines are toroidal, i.e. $B_{\text {tor }}^{\mu}$ is everywhere orthogonal to meridional $(r, \beta)$ planes through the $\hat{\alpha}$ axis. The non-zero Maxwell null tetrad component is

$$
\phi_{1}=\mathrm{i}\left(2 \sqrt{2} b_{0}\right) \Re^{-1} \digamma^{-2} .
$$

The relation $\Phi_{a b}=\phi_{a} \bar{\phi}_{b}$ between the Ricci $\Phi_{a b}$ and the Maxwell $\phi_{a}$ must be satisfied, and such is the case for the solution given here. This is seen explicitly since $\phi_{1} \bar{\phi}_{1}=\Phi_{11}$ given in equation (8). The Maxwell invariants are

$$
\begin{aligned}
I_{1}^{\text {tor }} & =\frac{1}{2} F^{\mu v} F_{\mu \nu}=-\left(\phi_{1}^{2}+\bar{\phi}_{1}^{2}\right)=16 \frac{b_{0}^{2}}{\mathfrak{R}^{2} \digamma^{4}} . \\
I_{2}^{\text {tor }} & =\frac{1}{2} F^{\mu v} F_{\mu \nu}^{*}=0 .
\end{aligned}
$$

The Weyl tensor vanishes on a particular torus. From equations (C.4) all Weyl components are zero at $\Re_{\mathrm{B}}^{4}=1 / b_{0}^{2}$, providing an effective 'magnetic range'. 


\section{Interior-exterior metric and extrinsic curvature}

\subsection{Boundary}

One can match interior metric $g^{\text {tor }}$ to exterior metric $g^{\text {tor-vac }}$. The boundary surface is located at

$$
r=r_{b}=\text { const. }
$$

The metric match is straight forward since $g^{\text {tor-vac }}$ is $g^{\text {tor }}$ with $b_{0}=0$. The second junction condition requires extrinsic curvatures to match at $r_{b}$. $\hat{N}^{\mu}$ is the unit normal at $r_{b}, \hat{N}_{\mu} \hat{N}^{\mu}=-1, \hat{N}_{\mu} \mathrm{d} x^{\mu}=N \mathrm{~d} r$,

$$
N=\mathfrak{R}^{2} \digamma\left[1+b_{0}^{2} \Re^{4} \sin ^{2} \beta(1+\digamma)\left(1+\digamma^{2}\right)\right]^{-1 / 2}, \quad N_{\text {vac }}=\mathfrak{R}^{2} .
$$

The extrinsic curvature, $K_{\mu \nu}$, is the projected covariant derivative

$$
K_{\mu \nu}^{\text {tor }}=\hat{N}_{\rho ; \sigma} \perp_{\mu}^{\rho} \perp_{\nu}^{\sigma}
$$

where $\perp_{\mu}^{\rho}=g_{\mu}^{\rho}+\hat{N}^{\rho} \hat{N}_{\mu}$ projects into the boundary.

\subsection{Interior}

At $r=r_{b}, K_{\mu \nu}^{\text {tor }}$, with $\Re_{b}=r_{0}+r_{b} \cos \beta$ and $\digamma_{b}=1+b_{0}^{2} \Re_{b}^{4}$, has components

$$
K_{(t)(t)}^{\mathrm{tor}}=-\frac{2 N_{b} \cos \beta\left(1+3 b_{0}^{2} \Re_{b}^{4}\right)}{\Re_{b} \digamma_{b}}
$$

$K_{(\alpha)(\alpha)}^{\mathrm{tor}}=-\frac{N_{b} \cos \beta\left(1-3 b_{0}^{2} \Re_{b}^{4}\right)}{\Re_{b}^{7} \digamma_{b}}$,

$K_{(\beta)(\beta)}^{\mathrm{tor}}=\frac{N_{b} r_{b}^{2} W_{b}}{\Re_{b} \digamma_{b}^{5}}$.

$$
\begin{aligned}
N_{b}=\Re_{b}^{2} \digamma_{b}[1 & \left.+b_{0}^{2} \Re_{b}^{4} \sin ^{2} \beta\left(1+\digamma_{b}\right)\left(1+\digamma_{b}^{2}\right)\right]^{-1 / 2} \\
W_{b}=3 \Re_{b} / r_{b} & -2 r_{0} / r_{b}+b_{0}^{2} \Re_{b}^{4}\left(3 \Re_{b} / r_{b}+2 r_{0} / r_{b}\right)-2 b_{0}^{4} \Re_{b}^{8}\left(4 \cos ^{3} \beta+9 \cos \beta-5 r_{0} / r_{b}\right) \\
& -\left(b_{0}^{6}+b_{0}^{10}\right) \Re_{b}^{4}\left(10 \Re_{b}^{5} / r_{b}-28 \Re_{b}^{4} \cos \beta \sin ^{2} \beta-3 \Re_{b} / r_{b}-2 r_{0} / r_{b}\right) \\
& +2 b_{0}^{8} \Re_{b}^{8}\left(5 \Re_{b} / r_{b}-14 \cos \beta \sin ^{2} \beta\right) .
\end{aligned}
$$

Relevant $g_{\text {tor }}^{\mu \nu}$ components are

$$
\begin{aligned}
& g_{\text {tor }}^{(t)}=\Re^{-4} \digamma^{-2}, \quad g_{\text {tor }}^{(\alpha)(\alpha)}=-\Re^{2} \digamma^{-2}, \\
& g_{\text {tor }}^{(\beta)(\beta)}=-r^{-2} \Re^{-4}\left(\digamma^{4} \sin ^{2} \beta+\cos ^{2} \beta\right)^{-1} .
\end{aligned}
$$

The trace of $K_{\mu \nu}^{\text {tor }}$ is

$$
K^{\text {tor }}=-\frac{N_{b}}{\Re_{b}^{5} \digamma_{b}^{3}}\left[2 \cos \beta\left(1+3 b_{0}^{2} \Re_{b}^{4}\right)-\cos \beta\left(1-3 b_{0}^{2} \Re_{b}^{4}\right)+\frac{W_{b}}{\digamma_{b}^{2}\left(\digamma_{b}^{4} \sin ^{2} \beta+\cos ^{2} \beta\right)}\right] .
$$




\subsection{Exterior}

$K_{\mu \nu}^{\text {tor-vac }}$ has components

$$
\begin{aligned}
& K_{(t)(t)}^{\mathrm{tor}-\mathrm{vac}}=-2 \Re_{b} \cos \beta, \quad K_{(\alpha)(\alpha)}^{\mathrm{tor}-\mathrm{vac}}=-\cos \beta / \Re_{b}^{5}, \\
& K_{(\beta)(\beta)}^{\mathrm{tor}-\mathrm{vac}}=r_{b} \Re_{b}\left(3 \Re_{b}-2 r_{0}\right) .
\end{aligned}
$$

Relevant $g_{\text {tor-vac }}^{\mu \nu}$ components are

The trace of $K_{\mu \nu}^{\text {tor-vac }}$ is

$$
g_{\text {tor-vac }}^{(t)(t)}=\Re^{-4}, \quad g_{\text {tor-vac }}^{(\alpha)(\alpha)}=-\Re^{2}, \quad g_{\text {tor-vac }}^{(\beta)(\beta)}=-r^{-2} \Re^{-4} .
$$

$$
K^{\mathrm{tor}-\mathrm{vac}}=-\frac{1}{\mathfrak{R}_{b}^{3}}\left(\cos \beta+3 \Re_{b} / r_{b}-2 r_{0} / r_{b}\right) .
$$

\section{Magnetic field match}

With unit normal $\hat{N}$, the magnetic field at the boundary requires

$$
\left(\vec{B}_{\text {tor }} \cdot \hat{N}\right)_{b}=\left(\vec{B}_{\text {vac }} \cdot \hat{N}_{\text {vac }}\right)_{b} \text {. }
$$

This condition is satisfied since the normal components are zero on both sides of the boundary. The tangential component must obey

$$
\left(\vec{B}_{\text {tor }} \times \hat{N}\right)_{b}=-\vec{J}_{\mathrm{s}},
$$

where $\vec{J}_{\mathrm{s}}$ is a surface current density. The jump in tangential component (non-zero to zero) requires an Israel surface layer to support $\vec{J}_{\mathrm{s}}$.

\subsection{Israel layer}

If the extrinsic curvatures do not match at the boundary, an Israel boundary layer is created. The stress-energy content of the Israel layer is constructed from the mismatch in the curvatures. The stress-energy of the boundary layer is [8]

$$
-8 \pi S_{v}^{\mu}=\left[K_{v}^{\mu}\right]-[K] \perp_{v}^{\mu} .
$$

Here $K=K_{\mu}^{\mu}$, and $[K]:=K^{\text {exterior }}-K^{\text {interior }}$ denotes the jump of $K$ (for any tensorial quantity defined on both sides of the boundary). The non-zero components of the stress-energy are $S_{(t)}^{(t)}, S_{(\alpha)}^{(\alpha)}$ and $S_{(\beta)}^{(\beta)}$. We find

$$
\begin{aligned}
& {\left[S_{(t)}^{(t)}\right]=-\frac{1}{\Re_{b}^{5} \digamma_{b}^{3}}\left[\Re_{b}^{2} \digamma_{b}^{3}\left(\cos \beta+2 r_{0} / r_{b}-3 \Re_{b} / r_{b}\right)+\frac{N_{b} W_{b}}{\digamma_{b}^{4} \sin ^{2} \beta+\cos ^{2} \beta}\right.}\left.-N_{b} \cos \beta\left(1-3 b_{0}^{2} \Re_{b}^{4}\right)\right] \\
& {\left[S_{(\alpha)}^{(\alpha)}\right]=\frac{1}{\Re_{b}^{5} \digamma_{b}^{3}}\left[\Re_{b}^{2} \digamma_{b}^{3}\left(2 \cos \beta+3 \Re_{b} / r_{b}-2 r_{0} / r_{b}\right)-\frac{N_{b} W_{b}}{\digamma_{b}^{4} \sin ^{2} \beta+\cos ^{2} \beta}\right.} \\
&\left.\quad-2 N_{b} \cos \beta\left(1+3 b_{0}^{2} \Re_{b}^{4}\right)\right] \\
& {\left[S_{(\beta)}^{(\beta)}\right]=\frac{\cos \beta}{\Re_{b}^{5} \digamma_{b}^{3}}\left[\Re_{b}^{2} \digamma_{b}^{3}-N_{b}\left(1+9 b_{0}^{2} \Re_{b}^{4}\right)\right] . }
\end{aligned}
$$

When the magnetic field is set to zero, i.e. $b_{0}^{2} \rightarrow 0$, all jumps vanish.

A graph of $S_{(t)}^{(t)}$ for weak magnetic field, with $\digamma_{b}^{2} \sim 1$, is bell-shaped and peaked at $(\beta)=\pi$ (figure 1). With large magnetic field, $\digamma_{b}^{2} \sim b_{0}^{4} \Re_{b}^{8}$, the graph has two bell-shaped 


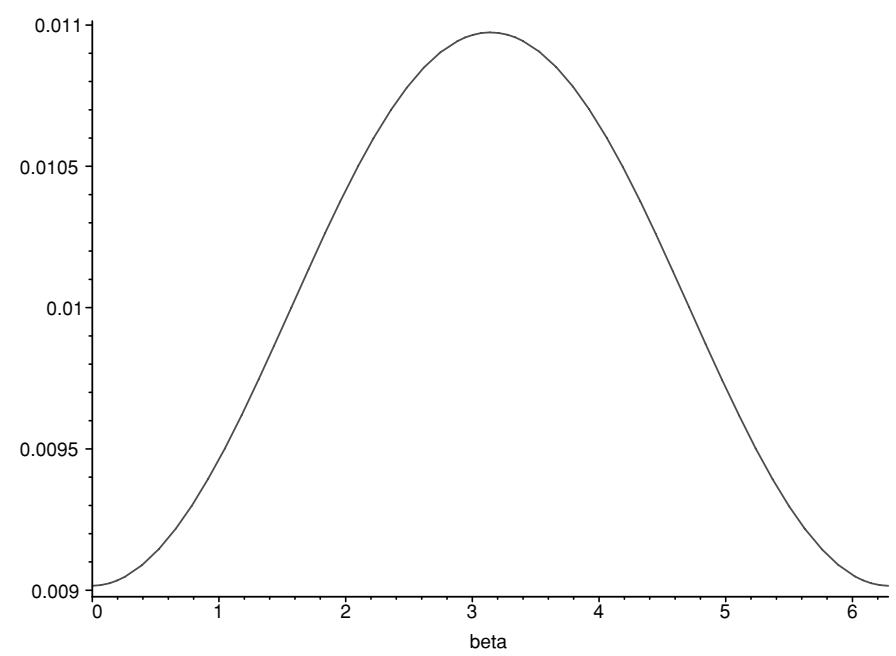

Figure 1. $r_{0} / r_{b}=10$. Weak magnetic field with $F_{b}^{2} \sim 1$. Vertical scale adjusted to graph.

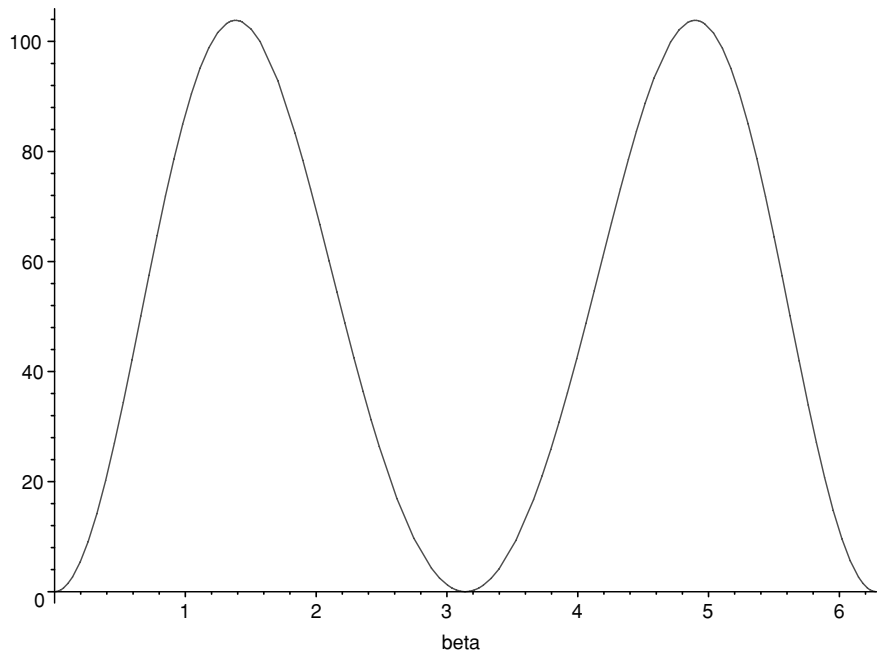

Figure 2. Large magnetic field. $F_{b}^{2} \sim b_{0}^{4} \Re_{b}^{8}$. Vertical scale adjusted to graph with $b_{0}=1$.

peaks at $(\beta)=\pi / 2,3 \pi / 2$ falling to zero at $(\beta)=\pi$ (figure 2 ). The other components have similar graphs. The stress-energy is peaked at the 'top, $\pi / 2$ ' and 'bottom, $3 \pi / 2$ ' of a given torus. Since magnetic energy subtracts quasilocally from mass energy, the stress-energy is greater at top and bottom to maintain the torus shape. With no magnetic energy, the stressenergy is a maximum at the innermost point, $(\beta)=\pi$, to balance mass attraction.

In the outer equatorial plane, with $\cos \beta=1$,

$$
\begin{aligned}
& {\left[S_{(t)}^{(t)}\right] \simeq b_{0}^{2}\left(1+r_{0} / r_{b}\right)\left(r_{b}-4 r_{0}\right)+O\left(r_{b}^{5}\right),} \\
& {\left[S_{(\alpha)}^{(\alpha)}\right] \simeq-2 b_{0}^{2}\left(1+r_{0} / r_{b}\right)\left(r_{b}-2 r_{0}\right)+O\left(r_{b}{ }^{2}\right),} \\
& {\left[S_{(\beta)}^{(\beta)}\right] \simeq-b_{0}^{2}\left(1+r_{0} / r_{b}\right)+O\left(r_{b}^{5}\right) .}
\end{aligned}
$$




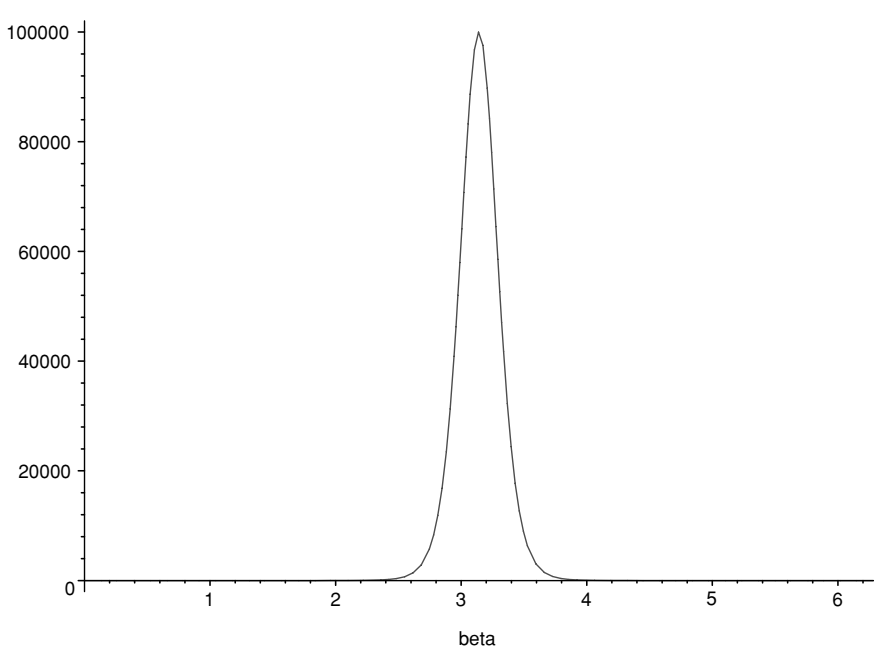

Figure 3. $J_{\mathrm{s}}$ versus $(\beta) . F_{b}^{2} \sim 1, r_{0} / r_{b}=1.1$ Vertical scale adjusted to graph.

Poisson [8] expresses the complete energy-momentum tensor as

$$
T_{\mu \nu}=\Theta(l) T_{\mu \nu}^{\text {exterior }}+\Theta(-l) T_{\mu \nu}^{\text {interior }}+\delta(l) S_{\mu \nu} .
$$

In this work $T_{\mu \nu}^{\text {exterior }}=0$ for $g^{\text {tor-vac }}$ and $T_{\mu \nu}^{\text {interior }}=T_{\mu \nu}^{\text {tor }}$ for $g^{\text {tor }}$. $\Theta$ is the Heaviside step function and $\delta(l)$ is a Dirac distribution of shell width $l$. We have

$$
T_{\mu \nu}=\Theta\left(r-r_{b}\right) T_{\mu \nu}^{\mathrm{tor}}+\delta\left(r-r_{b}\right) S_{\mu \nu} .
$$

The conservation equation for $S^{\mu}{ }_{\nu}$, its covariant divergence, expresses the jump in $T_{\mu \nu}^{\text {tor }}$ components at the boundary. To find the surface current density, we write the field tensor at the boundary

$$
F_{\text {interior }}^{\mu \nu}=\Theta\left(r-r_{b}\right) F_{\text {tor }}^{\mu \nu},
$$

where

$$
F_{\text {tor }}^{\mu \nu}=\left(\frac{4 b_{0}}{r \Re^{5} \digamma^{2}}\right)\left[\delta_{(r)}^{\mu} \delta_{(\beta)}^{v}-\delta_{(\beta)}^{\mu} \delta_{(r)}^{\nu}\right]
$$

$\nabla_{\nu} F_{\text {interior }}^{\mu \nu}=4 \pi J_{\mathrm{s}}^{\mu}=\delta\left(r-r_{b}\right) F_{\text {tor }}^{\mu(r)}$. With $\vec{J}_{\mathrm{s}}$ from equation (25) the current is

$$
4 \pi J_{\mathrm{s}}^{\mu} \partial_{\mu}=\delta\left(r-r_{b}\right)\left(\frac{4 b_{0}}{r_{b} \Re_{b}^{5} \digamma_{b}^{2}}\right) \partial_{\beta} .
$$

Any one of the current lines is an $\alpha=$ const circle around the torus. Together, the circles form a surface current on the bounding torus.

The graph of one of the current lines, $J_{\mathrm{s}}$ versus $(\beta)$, is bell-shaped and peaked at $(\beta)=\pi$. For weak magnetic field, with $\digamma_{b}^{2} \sim 1$, the shape is dominated by $\Re_{b}^{-5} \sim\left(r_{0} / r_{b}+\cos \beta\right)^{-5}$ (see figure 3). The curve narrows and the peak rises as $r_{b} \rightarrow r_{0}$, while the volume containing the magnetic field shrinks, with a spike as the volume nears zero. With large magnetic field, $\digamma_{b}^{2} \sim b_{0}^{4} \Re_{b}^{8}$, the current goes as $\Re_{b}^{-13}$. The curve shape is the same, with smaller magnitude. 


\section{Mass and energy}

\subsection{Magnetic energy}

The electromagnetic energy follows from integrating the magnetic energy density, $u$, over $t=$ const hypersurfaces within 3-volume $\sqrt{-g} d^{3} x=r \Re^{5} \digamma^{2} \mathrm{~d} \alpha \mathrm{d} \beta \mathrm{d} r$ :

$$
\begin{aligned}
u & =-\frac{1}{2} B_{\mu} B^{\mu} \\
& =8 b_{0}^{2} /\left(\Re^{2} \digamma^{4}\right) .
\end{aligned}
$$

The toroidal energy is

$$
U_{\text {tor }}(r)=2 b_{0}^{2} \int_{r_{0}}^{r} \int_{0}^{2 \pi} \frac{r^{\prime}\left(r_{0}+r^{\prime} \cos \beta\right)^{3}}{\left[1+b_{0}^{2}\left(r_{0}+r^{\prime} \cos \beta\right)^{4}\right]^{2}} \mathrm{~d} \beta \mathrm{d} r^{\prime} .
$$

Termwise integration provides

$$
U_{\text {tor }}(r)=\left[\frac{b_{0}^{2} r_{0}^{3}}{\left(1+b_{0}^{2} r_{0}^{4}\right)^{2}}\right]\left(r-r_{0}\right)^{2}+O\left(r-r_{0}\right)^{3} .
$$

\subsection{Komar mass}

The Komar superpotential, with Killing vector $k^{\mu}$, is

$$
\mathcal{U}_{\mathrm{komar}}^{\mu \nu}=\sqrt{-g}\left(\nabla^{\mu} k^{\nu}-\nabla^{\nu} k^{\mu}\right) \text {. }
$$

For $k^{\mu}=\delta_{(t)}^{\mu}$ we find

$$
\mathcal{U}_{\mathrm{komar}}^{\mu \nu}=b_{0}^{2} \frac{\Re^{2}}{\digamma}\left[r \cos \beta\left(\delta_{(t)}^{\mu} \delta_{(r)}^{\nu}-\delta_{(t)}^{\nu} \delta_{(r)}^{\mu}\right)-\sin \beta\left(\delta_{(t)}^{\mu} \delta_{(\beta)}^{\nu}-\delta_{(t)}^{\nu} \delta_{(\beta)}^{\mu}\right)\right] .
$$

The Komar mass is expressed by

$$
M\left(\partial_{t}\right)=\frac{1}{8 \pi} \oint \mathcal{U}_{\mathrm{komar}}^{\mu \nu} \mathrm{d} S_{\mu \nu}
$$

The mass integral, for $t=$ const, $r=$ const 2-surfaces with $\mathrm{d} S_{\mu \nu}=2\left(t_{, \mu} r_{, \nu}-t_{, \nu} r_{, \mu}\right) \mathrm{d} \alpha \mathrm{d} \beta$, is

$$
M_{\text {tor }}(r)=4 r \int_{0}^{2 \pi} \cos \beta\left[\frac{1+3 b_{0}^{2}\left(r_{0}+r \cos \beta\right)^{4}}{1+b_{0}^{2}\left(r_{0}+r \cos \beta\right)^{4}}\right] \mathrm{d} \beta .
$$

Integrating termwise provides the toroidal quasi-local mass

$$
M_{\text {tor }}(r)=\left[\frac{32 \pi b_{0}^{2} r_{0}^{3}}{\left(1+b_{0}^{2} r_{0}^{4}\right)^{2}}\right] r^{2}+O\left(r^{3}\right)
$$

The mass expansion is finite since vacuum starts at $r=r_{b}$.

\subsection{Sectional curvature mass}

Spherical fluids, with 2-metric $r^{2}\left(\mathrm{~d} \vartheta^{2}+\sin ^{2} \vartheta \mathrm{d} \varphi^{2}\right)$, have a well-defined sectional curvature mass given by

$$
-2 m_{\mathrm{sph}}(r)=r^{3} R_{\mu \nu \rho \sigma} \hat{\vartheta}^{\mu} \hat{\varphi}^{\nu} \hat{\vartheta}^{\rho} \hat{\varphi}^{\sigma}
$$

where the Riemann tensor is weighted by $r^{3}$ because of spherical geometry. The ReissnerNordström solution (Schwarzschild mass $m_{0}$, charge $q$ ) has sectional curvature mass

$$
2 m_{\mathrm{rn}}(r)=2 m_{0}-q^{2} / r .
$$

The electrostatic field energy due to charge $q$ has a negative quasilocal contribution. 
One can extend this notion to energy in tori. Vectors $\alpha^{\mu}$ and $\beta^{\mu}$ span $r=$ const tori and are surface-forming, i.e. $\beta^{v} \alpha_{; v}^{\mu}-\alpha^{v} \beta_{; v}^{\mu}=a \alpha^{\mu}+b \beta^{\mu}$. We define the torus sectional curvature mass as

$$
-2 m_{\text {tor }}(r)=\left[\Re^{6} R_{\mu \nu \rho \sigma} \hat{\alpha}^{\mu} \hat{\beta}^{\nu} \hat{\alpha}^{\rho} \hat{\beta}^{\sigma}\right]_{(\beta)=0}
$$

with the Riemann tensor weighted by $\mathfrak{R}^{6}$ because of the torus geometry in metric (4). Fixing $(\beta)=0$ selects $\Re=r_{0}+r$ for a given torus. We find

$$
m_{\text {tor }}(r)=m_{0} \frac{1-b_{0}^{2}\left(r_{0}+r\right)^{4}}{\left[1+b_{0}^{2}\left(r_{0}+r\right)^{4}\right]^{4}} .
$$

Parameter $m_{0}$ appears in the Riemann tensor when metric function $\digamma$ is scaled by $m_{0}$.

We see that the magnetic energy has a negative contribution to $m_{\text {tor }}$, just as the ReissnerNordström electrostatic energy. In the vacuum limit, $b_{0}^{2} \rightarrow 0, m_{\text {tor }}(r) \rightarrow m_{0}$.

\section{Complete metric}

Metrics $g^{\text {tor }}$ and $g^{\text {tor-vac }}$ combine to provide

$$
g^{\text {toroidal }}= \begin{cases}g^{\text {tor }}, & r_{0}<r \leqslant r_{b} \\ g^{\text {tor-vac }}, & r_{b} \leqslant r<\infty\end{cases}
$$

\subsection{Inner region}

The inner torus boundary has coordinates $\cos \beta=-1$ and $r=r_{0}$, with metric functions $\Re=0$ and $\digamma=1$. The toroidal metric goes as $g^{\text {tor }} \sim \Re^{4}\left(\mathrm{~d} t^{2}-\mathrm{d} r^{2}-r^{2} \mathrm{~d} \beta^{2}\right)-(1 / \Re)^{2} \mathrm{~d} \alpha^{2}$ and is not well behaved. Since $R^{\mu \nu} R_{\mu \nu}=\left(32 b_{0}^{2} / \mathfrak{R}^{2} \digamma^{4}\right)^{2}$ and $R_{\alpha \beta \mu \nu} R^{\alpha \beta \mu \nu} \sim 1 / \Re^{12}$, it is clear that $g^{\text {tor }}$ has a singularity at $r=r_{0}, \cos \beta=-1$, the $\Re=0$ loop. This reflects the singular axis of the original cylinder metric (1).

\subsection{Asymptotic region}

The vacuum region starts at $r_{b}$. For large distances $r \gg r_{0}$, and with $|\cos \beta| \simeq 1$, metric function $\Re=r_{0}+r \cos \beta$ go as $\Re \sim r$.

$g^{\text {tor }}$ is matched to a vacuum solution

$$
g_{\mu \nu}^{\text {tor-vac }} \mathrm{d} x^{\mu} \mathrm{d} x^{\nu}=\Re^{4}\left(\mathrm{~d} t^{2}-\mathrm{d} r^{2}-\Re^{-6} \mathrm{~d} \alpha^{2}-r^{2} \mathrm{~d} \beta^{2}\right) .
$$

Asymptotically, for $|\cos \beta|>0$,

$$
g^{\text {tor-vac }} \sim r^{4}\left(\mathrm{~d} t^{2}-\mathrm{d} r^{2}-r_{0}^{-6} \mathrm{~d} \alpha^{2}-r^{2} \mathrm{~d} \beta^{2}\right) .
$$

When $(\beta)$ is near $\pi / 2$ or $3 \pi / 2$ and $\cos \beta \sim \epsilon$ (near zero)

$$
g^{\text {tor-vac }} \sim \epsilon^{4} r^{4}\left(\mathrm{~d} t^{2}-\mathrm{d} r^{2}-r_{0}^{-6} \mathrm{~d} \alpha^{2}-r^{2} \mathrm{~d} \beta^{2}\right) .
$$

There are two directions which do not have a well-defined limiting metric: $(\beta)=\pi / 2,3 \pi / 2$, where the Gaussian curvature of the $(\alpha, \beta) 2$-surface is zero. This difficulty can be removed by choosing different torus coordinates. Morse and Feshbach [10] use torus coordinates with a hyperbolic radial coordinate. Present coordinates $\{r, \alpha, \beta\}_{\text {tor }}$ map to $\{\mu, \phi, \eta\}_{\mathrm{MF}}$. The analogue of $(\beta)$ is angle $(\eta)$. The Gaussian curvature of $(\phi, \eta) 2$-surfaces is

$$
\mathcal{K}_{\mathrm{MF}}=1-\cos \eta \cosh \mu
$$

and for $(\eta)=\pi / 2,3 \pi / 2, \mathcal{K}_{\mathrm{MF}}=1$. 
With $r^{4}$ as a conformal factor, $g^{\text {tor-vac }}$ can be compactified and is asymptotically flat. In the compactified diagram, the boundary of the singular cylinder curves from $I^{-}$to $I^{+}$while the null surfaces of the tori extend to $\mathcal{I}^{+}$. The spacetime of $g^{\text {tor }}$ is asymptotically flat and foliated by nested tori.

\section{Summary}

We have developed a method for mapping known static cylindrical magnetovac solutions to static solutions in torus coordinates. The cylinder topology has been changed from $R^{1} \times S^{1}$ to torus $S^{1} \times S^{1}$ by identifying the top and bottom cylinder ends. An explicit example has been presented with a toroidal magnetic field. The example satisfies Rainich conditions for valid electrovac solutions.

The new solution has three parameters: $r_{0}$ specifies the torus geometry, $b_{0}^{2}$ the magnetic field and magnetic energy density and $m_{0}$ is the sectional curvature mass parameter. The jump in magnetic field is supported by a surface current density in an Israel layer at the vacuum boundary. The solution has an asymptotically flat vacuum exterior, and an Einstein-Maxwell interior with a singular loop at the centre. By extending sectional curvature mass to include energy in tori, we have shown that the magnetic energy subtracts quasilocally from $m_{\text {tor }}$.

There are other exact cylinder solutions, such as the Melvin metric, which have no singular region but whose map to torus coordinates provide a more complicated magnetic field. These will be studied in future work, which will also add rotation to these solutions.

\section{Acknowledgments}

I am indebted to Professor Jean Krisch for reading and commenting on early versions of this work.

\section{Appendix A. Torus coordinates in Euclidean 3-space}

A torus can be generated by sweeping a circle, orthogonal to the $x-y$ plane, about the $z$-axis. In the $x-y$ plane $r_{0}$ is the distance from the $z$-axis to the centre of the generating circle, and $r$ is the radius of the generating circle. For ranges $0<r<\infty, 0<\alpha<2 \pi, 0<\beta<2 \pi$, with $\Re=r_{0}+r \cos \beta$,

$$
x=\Re \cos \alpha, \quad y=\Re \sin \alpha, \quad z=r \sin \beta .
$$

Cartesian and toroidal coordinates are related by

$$
x^{2}+y^{2}+z^{2}=\Re^{2}+r^{2} \sin ^{2} \beta .
$$

The torus $r=0$ lies in the $z=0$ plane and has circular radius $r_{0}$. Looking down the $z$-axis (about which $\alpha$ has range $0<\alpha<2 \pi$ ) at the torus, one sees the torus boundaries as two concentric circles. The $\beta=$ constant surfaces, $0<\beta<2 \pi$, are spheres centred on the $z$-axis. In the plane $\beta=\pi / 2, x^{2}+y^{2}+z^{2}=r_{0}^{2}+r^{2}$.

The Euclidean metric $\mathrm{d} x^{2}+\mathrm{d} y^{2}+\mathrm{d} z^{2}$ with coordinates (A.1) becomes

$$
\mathrm{d} r^{2}+\Re^{2} \mathrm{~d} \alpha^{2}+r^{2} \mathrm{~d} \beta^{2} .
$$

The $(\alpha, \beta) 2$-surface has Gaussian curvature

$$
\mathcal{K}=\frac{\cos \beta}{r\left(r_{0}+r \cos \beta\right)} .
$$


The angle $\beta$ ranges from 0 to $2 \pi$ over the generating circle, while $\mathcal{K}$ is negative, zero and positive.

$$
\begin{array}{ll}
\mathcal{K}<0 & \text { for } \quad \pi / 2<\beta<3 \pi / 2 \\
\mathcal{K}=0 & \text { for } \quad \beta=\pi / 2,3 \pi / 2 \\
\mathcal{K}>0 & \text { for } \quad 0<\beta<\pi / 2, \quad 3 \pi / 2<\beta<2 \pi .
\end{array}
$$

The Euler-Poincaré characteristic is

$$
\chi=\int_{0}^{2 \pi} \mathcal{K} r\left(r_{0}+r \cos \beta\right) \mathrm{d} \beta=0
$$

corresponding to the torus (sphere with one handle).

\section{Appendix B. Geodesics}

To better understand the region surrounding the central singularity we examine the geodesic flow. From metric $g^{\text {tor-vac }}$ we have a Lagrangean for geodesic paths

$$
\mathcal{L}=\frac{1}{2}\left(\Re^{4} \dot{t}^{2}-\Re^{4} \dot{r}^{2}-\Re^{-2} \dot{\alpha}^{2}-r^{2} \Re^{4} \dot{\beta}^{2}\right),
$$

where overdots denote $\mathrm{d} / \mathrm{d} s$. The geodesic equations follow from

$$
\frac{\mathrm{d}}{\mathrm{d} s} \frac{\partial \mathcal{L}}{\partial \dot{x}^{a}}-\frac{\partial \mathcal{L}}{\partial x^{a}}=0
$$

Killing symmetries $\partial_{t}$ and $\partial_{\alpha}$ yield first integrals

or

$$
\frac{\partial \mathcal{L}}{\partial \dot{t}}=E_{0}=\Re^{4} \dot{t}, \quad \frac{\partial \mathcal{L}}{\partial \dot{\alpha}}=-J_{0}=-\Re^{-2} \dot{\alpha}
$$

$$
\dot{t}=E_{0} / \Re^{4}, \quad \dot{\alpha}=J_{0} \Re^{2} .
$$

For $x^{a}=(\beta)$, the timelike geodesic equation is

$-r^{2} \mathfrak{R}^{4} \ddot{\beta}+2 r^{2} \mathfrak{R}^{3}(r \sin \beta) \dot{\beta}^{2}+2 \mathfrak{R}^{3}(r \sin \beta)\left(\dot{t}^{2}-\dot{r}^{2}\right)+\mathfrak{R}^{3}(r \sin \beta) \dot{\alpha}^{2}=0$.

If $(\beta)=\pi$ then $\ddot{\beta}=0$. Furthermore, if $\dot{\beta}=0$ and $\ddot{\beta}=0$, then the orbit remains in the $(\beta)=\pi$ plane. (In order to examine the singular region, we choose $(\beta)=\pi$ rather than $(\beta)=0$.) Again, from metric $g^{\text {tor-vac }}$

$$
\begin{aligned}
& 1=E_{0}^{2} / \Re^{4}-J_{0}^{2} \Re^{2}-\Re^{4} \dot{r}^{2}-r^{2} \Re^{4} \dot{\beta}^{2} \\
& 1=E_{0}^{2} /\left(r_{0}-r\right)^{4}-J_{0}^{2}\left(r_{0}-r\right)^{2}-\left(r_{0}-r\right)^{4} \dot{r}^{2} .
\end{aligned}
$$

We follow the central force problem of classical mechanics, where $E_{0}=\frac{1}{2} m \dot{r}^{2}+V_{\text {eff }}(r)$, and write $\dot{r}^{2}+V_{\text {eff }}(r)=$ const. Thus, the timelike geodesic paths in the $(\beta)=\pi$ plane can be described by the effective potential

$$
V_{\text {eff }}(r)=1 /\left(r-r_{0}\right)^{4}+J_{0}^{2} /\left(r-r_{0}\right)^{2}-E_{0}^{2} /\left(r-r_{0}\right)^{8} .
$$

For $J_{0}^{2}=E_{0}^{2}$, the graph of $V_{\text {eff }}$ has a hard negative core at $r=r_{0}$. The graph increases exponentially to positive values with a maximum at $r_{\max }$, and then falls to zero as $1 / r^{2} \cdot r_{\max }$ is a positive root of $J_{0}^{2}\left(r-r_{0}\right)^{6}+2\left(r-r_{0}\right)^{4}-4 E_{0}^{2}=0$. At $r=r_{\max }$ there is a closed, unstable, geodesic path around the singular region.

For $J_{0}^{2}=0$, the shape of the graph is the same, but the height of $r_{\max }$ is decreased. $r_{\text {max }}=r_{0}+\left(2 E_{0}^{2}\right)^{1 / 4}$.

For large angular momentum, $J_{0}^{2}>E_{0}^{2}$, the graph has no negative values (see figure 4). 


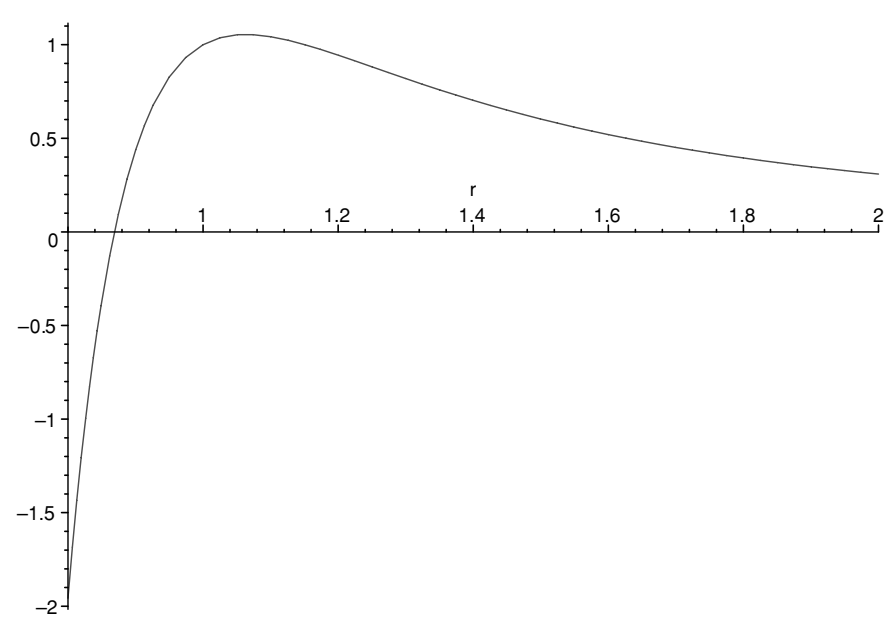

Figure 4. $V_{\text {eff }}$ with $r_{0}=0, J_{0}^{2}=E_{0}^{2}=1$.

\section{Appendix C. Torus null tetrad quantities}

The metric, $g_{\mu \nu}^{\text {tor }}=l_{\mu} n_{v}+n_{\mu} l_{\nu}-m_{\mu} \bar{m}_{v}-\bar{m}_{\mu} m_{v}$ with $\Re=r_{0}+r \cos \beta$ and $\digamma=1+b_{0}^{2} \Re^{4}$, has the null basis

$l_{\mu} \mathrm{d} x^{\mu}=\frac{\digamma}{\sqrt{2}}\left(\Re^{2} \mathrm{~d} t-\Re^{-1} \mathrm{~d} \alpha\right)$,

$n_{\mu} \mathrm{d} x^{\mu}=\frac{\digamma}{\sqrt{2}}\left(\Re^{2} \mathrm{~d} t+\Re^{-1} \mathrm{~d} \alpha\right)$,

$m_{\mu} \mathrm{d} x^{\mu}=-\frac{\mathfrak{R}^{2}}{\sqrt{2}}\left[\left(\digamma \cos \beta+\mathrm{i} \digamma^{-1} \sin \beta\right) \mathrm{d} r-\left(\digamma \sin \beta-\mathrm{i} \digamma^{-1} \cos \beta\right) r \mathrm{~d} \beta\right]$,

$\bar{m}_{\mu} \mathrm{d} x^{\mu}=-\frac{\mathfrak{R}^{2}}{\sqrt{2}}\left[\left(\digamma \cos \beta-\mathrm{i} \digamma^{-1} \sin \beta\right) \mathrm{d} r-\left(\digamma \sin \beta+\mathrm{i} \digamma^{-1} \cos \beta\right) r \mathrm{~d} \beta\right]$.

The spin coefficients are

$$
\begin{aligned}
& \epsilon=\sigma=\lambda=\rho=\mu=\gamma=0 \\
& \kappa=-\frac{3}{2 \sqrt{2}} \frac{1}{\Re^{3} \digamma} \\
& \nu=-\kappa \\
& \pi=\frac{1}{2 \sqrt{2}}\left(\frac{1}{\mathfrak{R}^{3} \digamma^{2}}\right)\left(1+9 b_{0}^{2} \Re^{4}\right) \\
& \tau=-\pi \\
& \alpha=-\frac{1}{\sqrt{2}}\left(\frac{1}{\mathfrak{R}^{3} \digamma^{2}}\right)\left(1-9 b_{0}^{2} \Re^{4}\right) \\
& \beta=-\alpha .
\end{aligned}
$$


$m_{\mu}$ and $\bar{m}_{\mu}$ are null geodesics, since $m_{\mu ; \nu} m^{\nu}=(\beta-\bar{\alpha}) m_{\mu}$. The only non-zero Ricci component is

$$
\Phi_{11}=8 \frac{b_{0}^{2}}{\Re^{2} \digamma^{4}} .
$$

The Weyl tensor null tetrad components are

$$
\begin{aligned}
& \Psi_{0}=-\left(\frac{3}{\mathfrak{R}^{6} \digamma^{3}}\right)\left(1-b_{0}^{2} \Re^{4}\right) \\
& \Psi_{1}=\Psi_{3}=0 \\
& \Psi_{2}=-\left(\frac{1}{\mathfrak{R}^{6} \digamma^{4}}\right)\left(1-b_{0}^{2} \Re^{4}\right)\left(1+9 b_{0}^{2} \Re^{4}\right) \\
& \Psi_{4}=\Psi_{0}
\end{aligned}
$$

which correspond to Petrov type $\mathbf{I}$.

The quadratic Ricci and Weyl invariants are

$$
\begin{aligned}
& R^{\mu \nu} R_{\mu \nu}=\left(32 b_{0}^{2} / \Re^{2} \digamma^{4}\right)^{2}, \\
& C^{\alpha \beta \mu \nu} C_{\alpha \beta \mu \nu}=\frac{192}{\Re^{12} \digamma^{4}} \frac{\left(1-b_{0}^{2} \Re^{4}\right)\left(1+6 b_{0}^{2} \Re^{4}+21 b_{0}^{4} \Re^{8}\right)}{\left(1+6 b_{0}^{2} \Re^{4}+b_{0}^{4} \Re^{8}\right)} .
\end{aligned}
$$

\section{Appendix D. Relativistic electromagnetic field}

The Maxwell field is represented by skew tensor $F_{\mu \nu}$, with dual field $F_{\mu \nu}^{*}=\frac{1}{2} \sqrt{-g} \varepsilon_{\mu \nu \alpha \beta} F^{\alpha \beta}$. The symmetric energy-momentum is

$$
T_{\mu \nu}=\frac{1}{4 \pi}\left(F_{\mu \alpha} F_{\nu}^{\alpha}+\frac{1}{4} g_{\mu \nu} F_{\alpha \beta} F^{\alpha \beta}\right) .
$$

In a local Lorentz frame, the energy density is

$$
T_{00}=-\left(E^{2}+B^{2}\right) / 8 \pi \text {. }
$$

The Einstein field equations provide the trace-free Ricci tensor

$$
R_{\mu \nu}=-\left(F_{\mu \alpha} F_{v}^{\alpha}+\frac{1}{4} g_{\mu \nu} F_{\alpha \beta} F^{\alpha \beta}\right) .
$$

For static systems there exists a hypersurface orthogonal timelike Killing vector $\xi^{\mu} \partial_{\mu}=\partial_{t}$. The constant time hypersurfaces form a family of Lorentz frames with unit normal $\hat{t}^{v}=$ $\xi^{\nu} /\left(\xi_{\mu} \xi^{\mu}\right)^{1 / 2}$. Local electric and magnetic fields are defined by

$$
E_{\mu}=F_{\mu \nu} \hat{t}^{\nu}=(0, \vec{E}), \quad B_{\mu}=F_{\mu \nu}^{*} \hat{t}^{\nu}=(0,-\vec{B}) .
$$

Maxwell's static equations are

$$
\nabla \cdot \vec{E}=4 \pi \rho, \quad \nabla \times \vec{E}=0, \quad \nabla \cdot \vec{B}=0, \quad \nabla \times \vec{B}=4 \pi \vec{J}
$$

The field invariants are

$$
I_{1}:=\frac{1}{2} F_{\mu \nu} F^{\mu \nu}=B^{2}-E^{2}, \quad I_{2}:=\frac{1}{2} F^{\mu \nu} F_{\mu \nu}^{*}=-2 E_{\mu} B^{\mu} .
$$

The Maxwell components on an anti-self dual bivector basis are

$$
\frac{1}{2}\left(F^{\mu \nu}+\mathrm{i} F^{* \mu \nu}\right)=\phi_{0} U^{\mu \nu}-\phi_{1} M^{\mu \nu}+\phi_{2} V^{\mu \nu}
$$


The field invariants are

$$
I_{1}+\mathrm{i} I_{2}=4\left(\phi_{0} \phi_{2}-\phi_{1}^{2}\right)
$$

There are three necessary and sufficient Rainich conditions [11] for $T_{\mu \nu}$ to be an electrovac energy-momentum tensor.

(1) $T_{\mu \nu}$ (and Ricci) must be trace-free.

$$
g^{\mu \nu} T_{\mu \nu}=g^{\mu \nu} R_{\mu \nu}=0 .
$$

(2) The 'square' of $T_{\mu \nu}$ is a positive multiple of the unit tensor.

$$
\begin{aligned}
& T_{\alpha}^{\mu} T_{v}^{\alpha}=\left(I_{1}^{2}+I_{2}^{2}\right) \delta_{v}^{\mu} \\
& \text { or } R_{\alpha}^{\mu} R_{v}^{\alpha}=\frac{1}{4}\left(R_{\alpha \beta} R^{\alpha \beta}\right) \delta_{v}^{\mu} .
\end{aligned}
$$

(3) Since the electromagnetic energy-momentum density, $T_{00}$, is negative in a local Minkowski frame, it is necessary for any timelike vector $t^{\mu}$ that

$$
T_{\mu \nu} t^{\mu} t^{\nu} \leqslant 0
$$

\section{References}

[1] Ferrario L and Wickramasinghe D T 2005 Mon. Not. R. Astron. Soc. 356615

[2] Shapiro S L and Teukolsky S A 1983 Black Holes, White Dwarfs, and Neutron Stars (New York: Wiley) chapter 7

[3] Ioka K and Sasaki M 2004 Astrophys. J. 600296

[4] Cutler C 2002 Phys. Rev. D 66084025

[5] do Carmo M P 1976 Differential Geometry of Curves and Surfaces (New Jersey: Prentice-Hall) p 157

[6] Israel W 1977 Phys. Rev. D 15935

[7] Kramer D, Stephani H, Herlt E, MacCallum M and Schmutzer E (ed) 2003 Exact Solutions of Einstein's Field Equations 2nd edn (Cambridge: Cambridge University Press)

[8] Poisson E 2004 A Relativist's Toolkit (Cambridge: Cambridge University Press) p 89

[9] Jackson J D 1999 Classical Electrodynamics 3rd edn (New York: Wiley)

[10] Morse P M and Feshbach H 1953 Methods of Theoretical Physics part II (New York: McGraw Hill) chapter 10

[11] Witten L 1962 A geometric theory of the electromagnetic and gravitational fields Gravitation ed L Witten (New York: Wiley) 